\title{
TOA Estimation Improvements in Multipath Environments by Measurement Error Models
}

Andreas Bergström, Gustaf Hendeby, Fredrik Gunnarsson and Fredrik Gustafsson

\section{Book Chapter}

N.B.: When citing this work, cite the original article.

Part of: Proceedings of the 2017 IEEE 28th Annual International Symposium on Personal, Indoor, and Mobile Radio Communications (PIMRC), 2017, pp. 1.8. ISBN: 9781538635315 (Article), 9781538635308 (USB)

Series: Annual International Symposium on Personal, Indoor, and Mobile Radio Communications (PIMRC), ISSN 2166-9589, 2017

\section{DOI: https:// doi.org/ 10.1109/PIMRC.2017.8292377}

Copyright: Institute of Electrical and Electronics Engineers (IEEE)

Available at: Linköping University Institutional Repository (DiVA)

http:// urn.kb.se/ resolve?urn=urn:nbn:se:liu:diva-142760 


\title{
TOA Estimation Improvements in Multipath Environments by Measurement Error Models
}

\author{
Andreas Bergström*†, Gustaf Hendeby*, Fredrik Gunnarsson*† and Fredrik Gustafsson* \\ ${ }^{*}$ Department of Electrical Engineering, Linköping University, Linköping, Sweden, \\ Email: \{fistname.lastname $\}$ liu.se \\ ${ }^{\dagger}$ Ericsson Research, Linköping, Sweden, Email: \{andreas.a.bergstrom, fredrik.gunnarsson\}@ericsson.com
}

\begin{abstract}
Many positioning systems rely on accurate time of arrival measurements. In this paper, we address not only the accuracy but also the relevance of Time of Arrival (TOA) measurement error modeling. We discuss how better knowledge of these errors can improve relative distance estimation, and compare the impact of differently detailed measurement error information. These models are compared in simulations based on models derived from an Ultra Wideband (UWB) measurement campaign. The conclusion is that significant improvements can be made without providing detailed received signal information but with a generic and relevant measurement error model.
\end{abstract}

\section{INTRODUCTION}

Positioning is a key component in many applications, ranging from crude to very accurate positioning. The increasing interest in Internet of Things (IoT) also brings requirements for ubiquitous connectivity and positioning. Positioning support in terrestrial cellular networks is mainly driven by regulatory requirements for emergency calls. However, the considered methods are also generally applicable to other use cases. Positioning in wireless networks can also be based on dedicated signals, for example to estimate the range between a device and a wireless node. For an overview of different methods, see [1-3]. Much research has been focused on positioning using UWB ranging, partly because of the attractive properties of fine time and multipath resolution, but also because of the equipment available for experiments. An overview of UWB positioning is provided in [4].

Several positioning methods in wireless networks are based on TOA estimation of a received signal with known characteristics. A simple strategy is to estimate the TOA as the arrival time of the strongest crosscorrelation peak among the multiple detected peaks. An alternative is to consider all peaks above a threshold relative the strongest peak, since the latter is not always is the first peak etc. Time of arrival estimation is further analyzed in different contexts in [5-8].

Given a mechanism for TOA estimation, it is possible to consider several different positioning methods:

Downlink TOA When the transmission time is encoded in the transmitted signals, it is possible to determine the Time of Flight (ToF) of the signals from transmitter to

978-1-5386-3531-5/17/\$31.00 @ 2017 IEEE receiver, as for example in Global Navigation Satellite Systems (GNSS).

Downlink TDOA When the transmission time is unknown, one option is Time Difference of Arrival (TDOA), where a device/entity reports the time difference of a received signal and a reference time. Typically in downlink TDOA, the latter is the reception time of a reference cell, and in uplink TDOA, it is configurable.

Ranging When the transmission time is unknown, two nodes can exchange packets with information about processing time to enable a receiver to estimate the round-trip time (RTT) as twice the ToF. RTT is typically the basis in UWB positioning, but is also a component in WiFi positioning based on Fine Timing Measurements (FTM) and cellular time alignment.

In this paper, we investigate range estimation based of TOA estimates, in particular with a focus on TOA estimation error modeling. We consider data from a UWB measurement campaign to discuss relevant such TOA estimation error models, and use the derived models for simulations and evaluations. In particular, we investigate different ways to accommodate information about the measurement errors including the possibility of false detection of noise peaks and missing the line of sight component and selecting a later non-line of sight peak. We see that, as long as all relevant peaks are covered by the measurement error model, we get significant improvements even with a rather generic model.

The rest of this paper is organized as: Sec. II presents how TOA measurements can be modeled taking into account more details of the radio environment, whereafter Sec. III proposes a practical way of performing these TOA measurements using a RTT procedure. As discussed in Sec. IV, errors may arise during the measurement and estimation procedure. The receiver knowledge of these errors is then discussed and put in a Bayesian filtering context in Sec. V. In Sec. VI, a UWB measurement campaign is performed in order to support and concretizise the earlier derived channel and error models with real measurement data. These models are then used in the simulations for which the results are presented and discussed in Sec. VII, followed by the final conclusions in Sec. VIII. 


\section{TOA Measurements}

\section{A. Basic Modeling}

First, a signal $\mathbf{u}(t)$ is sent by the transmitter, whereafter the receiver will observe a received signal $\mathbf{y}(t)$. This can, following the methodology of [9], be modeled as a Linear Time-Varying (LTV) system as in Fig. 1. Here, the receive antenna observation time is $t$, the time delay in the channel $\tau$, the (complex baseband) input $\mathbf{u}(t)$, channel impulse response $\mathbf{H}(t, \tau)$ and noise $\mathbf{e}(t) \sim \mathcal{C N}(0, \Sigma)$.

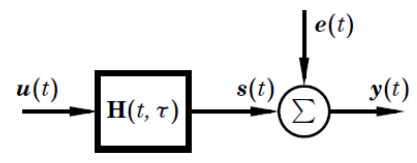

Fig. 1. Model of the radio channel as a LTV system.

Assuming the channel to be time-invariant as well as non-dispersive, we can write $\mathbf{H}(t, \tau)=\mathbf{H}_{t, \tau} \delta\left(t-\tau_{D P}\right)$, wherein $\mathbf{H}_{t, \tau}$ is the channel matrix, $\delta(t)$ is the Dirac delta function and $\tau_{D P}$ is how much the Direct Path (DP) is delayed by this transmission. The DP represents the shortest path (in a Euclidean sense) the signal can traverse between transmitter and receiver, and is hence used for TOF estimation. We thereby get

$$
\mathbf{y}(t)=\mathbf{H}_{t, \tau} \star \mathbf{u}\left(t-\tau_{D P}\right)+\mathbf{e}(t) .
$$

where ' $\star$ ' means convolution. If $\mathbf{u}(t)$ is known by the receiver, we can estimate $\mathbf{H}(t, \tau)$ and $\tau_{D P}$ ([3], [10], [11]).

In a cellular network with one UE and $M$ BSs, we let $\tau_{D P}^{i}(t)$ be the estimated TOF for the DP between BS $i=1, \ldots, M$ and the UE, which hence gives

$$
\tau_{D P}^{i}(t)=\frac{1}{c}\left\|p^{i}-x(t)\right\|_{2}+e^{i}(t),
$$

in which $x(t)$ is the UE location, $p^{i}$ is the BS $i$ location, $c=c_{0} / 1.0003$ is the speed of light in air and the noise is zero-mean Gaussian, i.e. $e^{i}(t) \sim p_{E}^{i}=\mathcal{N}\left(0,\left(\sigma_{E}^{i}\right)^{2}\right)$. We can now estimate the UE location $x(t)$ as

$$
\hat{x}(t)=\arg \min _{x} \underbrace{\sum_{i=i}^{M} p_{E}^{i}\left(\tau_{D P}^{i}(t)-\frac{1}{c}\left\|p^{i}-x(t)\right\|_{2}\right)}_{\text {Loss function for TOA }} .
$$

\section{B. Multipath Aspects}

In many situations, the signal $\mathbf{u}(t)$ will be subject to multipath propagation due to scattering and/or reflections in the environment. The signal is essentially split into a number of Multi-Path Components (MPCs), as per Fig. 2. The channel matrix $\mathbf{H}_{t, \tau}$ can be decomposed as

$$
\mathbf{H}_{t, \tau}=\sum_{r=1}^{N_{r}} \mathbf{H}_{t, \tau}^{(r)}
$$

where $\mathbf{H}_{t, \tau}^{(r)}$ is the channel matrix for the $r$ th MPC.

The TOF $\tau$ can be seen as originating from an underlying statistical distribution where each peak corresponds

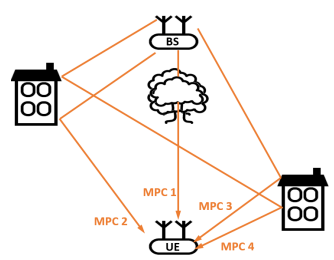

(a)

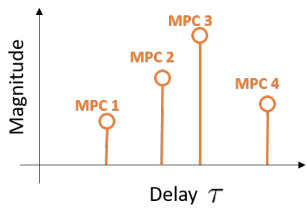

(b)
Fig. 2. Illustration of (a) multi-path propagation and (b) the resulting delays $\tau$ and magnitude of the received signal for each MPC.

to one of the MPCs. The spread of the peaks relates to both additional fine level scattering having occurred for each MPC, as well as receiver measurement noise. In this paper, we model $\tau$ for the $N_{r}$ MPCs relating to BS $i$ as a weighted Gaussian variable

$$
\tau_{r}^{i}(t) \sim w_{r}^{i} \mathcal{N}\left(\mu_{r}^{i},\left(\sigma_{r}^{i}\right)^{2}\right) \text { for } r=1, \ldots, N_{r} .
$$

It should be noted that this distribution need not be Gaussian, but could in many cases be modeled using other, potentially skewed, distributions [12]. Here we will however treat it as a stationary Gaussian distribution as per above. The parameters are estimated in Sec. VI.

\section{RTT ESTIMATION}

So far, we have considered only the TOA measurements and MPCs in one direction. In practice however, the corresponding TOA measurements and MPCs in both directions should be considered as depicted in Fig. 3 (to be compared with Fig. 2). We may hence, in analogy with (2) and (3), form

$$
\begin{aligned}
\tau_{d}^{i, D P_{d}}(t) & =\frac{1}{c}\left\|p^{i}-x(t)\right\|_{2}+e_{d}^{i}(t), \\
\tau_{u}^{i, D P_{u}}(t) & =\frac{1}{c}\left\|p^{i}-x(t)\right\|_{2}+e_{u}^{i}(t),
\end{aligned}
$$

where $e_{d}^{i}(t) \sim p_{E_{d}}^{i}=\mathcal{N}\left(0,\left(\sigma_{E_{d}}^{i}\right)^{2}\right)$ and $e_{u}^{i}(t) \sim p_{E_{u}}^{i}=$ $\mathcal{N}\left(0,\left(\sigma_{E_{u}}^{i}\right)^{2}\right)$. It should be noted that in the general case, $p_{E_{d}}^{i} \neq p_{E_{u}}^{i}$ since e.g. uplink and downlink may be operating in different frequency bands, there may be different hardware involved in the UE and/or BSs for the respective directions, there can be differences in-between different base stations etc.

Now, measuring $\tau_{d}^{i, D P_{d}}(t)$ and $\tau_{u}^{i, D P_{u}}(t)$ is not entirely straightforward, since direct measurements thereof would put strict requirements on either synchronization between the BS and the UE or alternatively force an

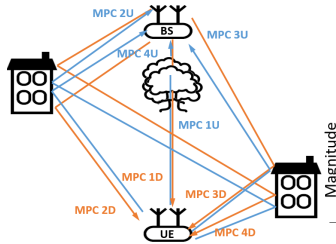

(a)

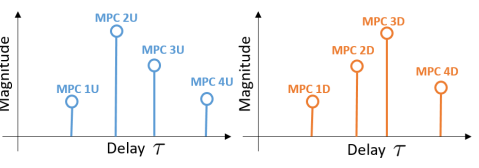

(b)
Fig. 3. Same as Fig. 2, but now including both DL and UL. 


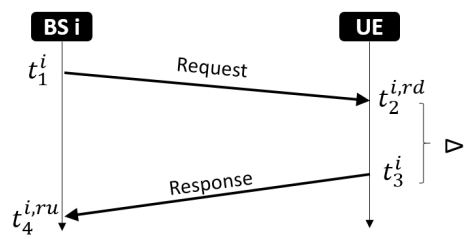

Fig. 4. Illustration of the proposed RTT measurement based method.

estimation of the synchronization offset between them ([10], [3]). Instead, we here propose a RTT measurement based method, as in Fig. 4, where we let $t_{1}^{i}$ be the time when a known DL signal is transmitted from BS $i$. This is received by the UE at times $t_{2}^{i, r_{d}}$ for $r_{d}=1, \ldots, N_{r}^{d}$, i.e. one per DL MPC. The UE has a known processing delay $\Delta$. At time $t_{3}^{i}$, the UE transmits an UL signal, which is received by the BS at times $t_{4}^{i, r_{u}}$ for $r_{u}=1, \ldots, N_{r}^{u}$, i.e. one per UL MPC.

Assuming that the DP is correctly identified in both directions, i.e. $r_{d}=D P_{d}$ and $r_{d}=D P_{u}$, this allows us to measure the RTT $R^{i}(t)$ based on Fig. 4 as

$$
\begin{aligned}
R^{i}(t) & =t_{4}^{i, D P_{u}}-t_{1}^{i}=t_{4}^{i, D P_{u}}-t_{3}^{i}+\Delta+t_{2}^{i, D P_{d}}-t_{1}^{i} \\
& =\tau_{d}^{i, D P_{d}}(t)+\tau_{u}^{i, D P_{u}}(t)+\Delta \\
& =\frac{2}{c}\left\|p^{i}-x(t)\right\|_{2}+\Delta+\underbrace{e_{d}^{i}(t)+e_{u}^{i}(t)}_{e_{d+u}^{i}(t)}
\end{aligned}
$$

in which $e_{d+u}^{i}(t) \sim p_{E_{d+u}}^{i}=p_{E_{d}}^{i} \star p_{E_{u}}^{i}$. Assuming these variables to be mutually uncorrelated we get $p_{E_{d+u}}^{i}=\mathcal{N}\left(0,\left(\sigma_{E_{d}}^{i}\right)^{2}+\left(\sigma_{E_{u}}^{i}\right)^{2}\right)$. In analogy with (3), we can now estimate the UE location as

$$
\hat{x}(t)=\arg \min _{x} \underbrace{\sum_{i=i}^{M} p_{E_{d+u}}^{i}\left(R^{i}(t)-\Delta-\frac{2}{c}\left\|p^{i}-x(t)\right\|_{2}\right)}_{\text {Loss function for RTT Estimated TOA }} .
$$

\section{ERror Model}

So far, we have assumed that the DP was always correctly identified by both BS and UE. This is an idealized assumption since, as discussed in [5-7, 11, 13, 14], it may e.g. happen that (as in Fig. 2) the DP (i.e. MPC 1) is obscured, so that it is not the strongest, and may even be hard to detect. Any measurement based on a non-DP (e.g. MPC 2, 3 or 4) will cause an over-estimation of the range between UE and BS. This is referred to as a Late Detection (LD) error. Also, noise may be erroneously interpreted as MPCs $[5,11]$, potentially with a shorter delay than the DP and hence under-estimating the range between UE and BS. This is referred to as a False Alarm (FA) error. These both errors are illustrated in Fig. 5.

We here model the FA error $e_{F A}^{i}(t)$ as belonging to the uniform distribution $p_{E_{F A}}^{i}$, i.e.

$$
e_{F A}^{i}(t) \sim p_{E_{F A}}^{i}=\mathcal{U}\left(-\frac{1}{2} \mu_{D P}^{i}, 0\right) .
$$

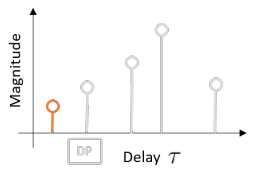

(a) False Alarm (FA)

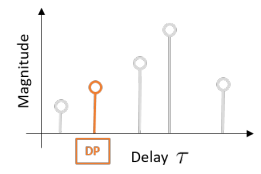

(b) Correct

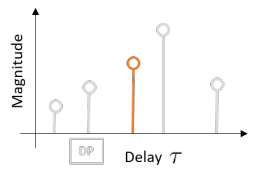

(c) Late Detection (LD)
Fig. 5. Potential error cases where 'DP' indicates the true DP, and the colored bar what the receiver believes to be the DP. The cases are: (a) False Alarm (FA) where noise is misinterpreted as the DP so that $\tau<\tau_{D P}$, (b) Correct behavior where the DP is correctly interpreted so that $\tau=\tau_{D P}$ and (c) Late Detection (LD) where the DP is misinterpreted as noise so that $\tau>\tau_{D P}$.

We further model the LD error $e_{L D}^{i}(t)$ as belonging to the distribution $p_{E_{L D}}$ which is the weighted Gaussian mixture consisting of all MPCs, except for the DP, i.e.

$$
e_{L D}^{i}(t) \sim p_{E_{L D}}=\sum_{\substack{r=1 \\ r \neq D P}}^{N_{r}} w_{r}^{i} \mathcal{N}\left(\mu_{r}^{i}-\mu_{D P}^{i}, \sigma_{r}^{i}\right) .
$$

By associating these both errors with the fixed probabilities of $P_{F A}$ and $P_{L D}$ respectively, (6) now becomes

$$
\begin{aligned}
\tau_{d}^{i, D P_{d}}(t)=\frac{1}{c}\left\|p^{i}-x(t)\right\|_{2}+\tilde{e}_{d}^{i}(t), & \tilde{e}_{d}^{i}(t) \sim p_{\tilde{E}_{d}}^{i} \\
\tau_{u}^{i, D P_{u}}(t)=\frac{1}{c}\left\|p^{i}-x(t)\right\|_{2}+\tilde{e}_{u}^{i}(t), & \tilde{e}_{u}^{i}(t) \sim p_{\tilde{E}_{u}}^{i}
\end{aligned}
$$

for which the distributions $p_{\tilde{E}_{d}}^{i}$ and $p_{\tilde{E}_{u}}^{i}$ are given by

$$
\begin{aligned}
& p_{\tilde{E}_{d}}^{i}=\left(1-P_{F A}-P_{L D}\right) p_{E_{d}}^{i}+P_{F A} p_{E_{F A}}^{i}+P_{L D} p_{E_{L D}}^{i} \\
& p_{\tilde{E}_{u}}^{i}=\left(1-P_{F A}-P_{L D}\right) p_{E_{u}}^{i}+P_{F A} p_{E_{F A}}^{i}+P_{L D} p_{E_{L D}}^{i} .
\end{aligned}
$$

Further, the RTT estimate of (7) similarly becomes

$$
R^{i}(t)=\frac{2}{c}\left\|p^{i}-x(t)\right\|_{2}+\Delta+\tilde{e}_{d+u}^{i}(t)
$$

for which the resulting error $\tilde{e}_{d+u}^{i}(t)$ is given by

$$
\tilde{e}_{d+u}^{i}(t) \sim p_{\tilde{E}_{d+u}}^{i}=p_{\tilde{E}_{d}}^{i} \star p_{\tilde{E}_{u}}^{i}
$$

No explicit expressions of these distributions are given here, but instead numerically later in Sec. VI.

\section{Estimation Model}

The simulations in this paper will, for the sake of clarity and simplicity, be performed for one UE and one single BS, i.e. $M=1$. We will hence estimate the distance between UE and BS, rather than the actual 2D or 3D position of the UE.

The estimation is done in a Bayesian frameworkmore precisely using a Sequential Importance Resampling (SIR) Particle Filter (PF) ([10, 15]). The number of particles is $N=100$ and resampling is performed when $n_{\text {eff }} \leq 2 / 3 N$. 


\section{A. Motion Model}

Letting the state $x(t) \in \mathbb{R}$ be the Euclidean distance between UE and BS, we use a random walk model

$$
x(t+T)=x(t)+T v(t)
$$

given a sample time $T$, a prior $x(0) \sim \mathcal{N}\left(\mu_{0}, \sigma_{0}^{2}\right)$ and process noise $v(t) \sim \mathcal{N}\left(0, q_{1}^{2}\right)$. A more advanced model is clearly possible [10], but for the sake of simplicity and clarity, we stick with this simple model. In this paper we use $T=0.1 \mathrm{~s}, q_{1}=1 \mathrm{~m} / \mathrm{s}, \mu_{0}=8 \mathrm{~m}$ and $\sigma_{0}=1 \mathrm{~m}$.

\section{B. Measurement Model}

The simplest measurement model is based on a single direction measurements in (11). Since $x(t)$ already represents the distance between UE and BS, we get the measured delay in either direction (hence we drop the $d$ and $u$ subscripts) as

$$
y^{i}(t)=\tau^{i, D P}(t)=\frac{1}{c} x(t)+g_{\tau}^{i}(t)
$$

wherein $g_{\tau}^{i}(t)$ is the assumed error model. We further define a model based on the RTT measurement of (13)

$$
y^{i}(t)=R^{i}(t)=\frac{2}{c} x(t)+g_{R}^{i}(t)
$$

wherein $g_{R}^{i}(t)$ is the assumed error model in this case.

Now, we define four different models of the errors $g_{\tau}^{i}(t)$ and $g_{R}^{i}(t)$, depending on how well-informed the receiver is with respect to the errors described in Sec. IV as listed below.

Model A Perfect DP identification is assumed, i.e.

$$
\begin{aligned}
g_{\tau}^{i}(t) & =e^{i}(t) \sim p_{E}^{i} & \text { from (2) and (6) } \\
g_{R}^{i}(t) & =e_{d+u}^{i}(t) \sim p_{E_{d+u}}^{i} & \text { from (7) }
\end{aligned}
$$

Model B Perfect DP identification is assumed, but we increase the variance with a factor 10 in the single direction case and 20 in the RTT case.

$$
\begin{aligned}
g_{\tau}^{i}(t) & \sim \mathcal{N}\left(0,10\left(\sigma_{E}^{i}\right)^{2}\right) \\
g_{R}^{i}(t) & \sim \mathcal{N}\left(0,20\left(\sigma_{E}^{i}\right)^{2}\right)
\end{aligned}
$$

Model C Simplifying the true error models by modeling the LD error approximately as belonging to a uniform distribution on the interval spanned by the Gaussian mixture, i.e.

$$
\hat{e}_{L D}^{i}(t) \sim p_{\hat{E}_{L D}}=\mathcal{U}\left(0, \mu_{r M A X}^{i}+3 \sigma_{r M A X}^{i}\right)
$$

where $r^{M A X}$ is the MPC with the largest expected mean delay, i.e. $r^{M A X}=\max _{r} \mu_{r}$. This is then used in place of $e_{L D}^{i}(t)$ in (11) through (14).

Model D The true error models (11), (13) are assumed known, i.e.

$$
\begin{aligned}
g_{\tau}^{i}(t) & =\tilde{e}^{i}(t) \sim p_{\tilde{E}}^{i} \\
g_{R}^{i}(t) & =\tilde{e}_{d+u}^{i}(t) \sim p_{\tilde{E}_{d+u}}^{i}
\end{aligned}
$$

Again, no explicit expressions of these distributions are given here, but instead numerically later in Sec. VI.

\section{Vi. Measurement Campaign}

In this section, we fill the models of Sec. II to $\mathrm{V}$ with data. For this, UWB measurements have been conducted using the BeSpoon academic kit [16] with one SpoonPhone ${ }^{\mathrm{TM}}$ (which represents the UE) and one tag (which represents the BS). The measurements where performed in a typical office environment with the SpoonPhone ${ }^{\mathrm{TM}} / \mathrm{UE}$ at two distinct locations, here referred to as the 'LoS Scenario' and the 'NLoS Scenario' respectively, as shown in Fig. 6. The measurements were performed with a sampling rate of $4 \mathrm{~Hz}$ during approx. $20 \mathrm{~min}$, hence $\sim 4800$ measurements were collected in total.

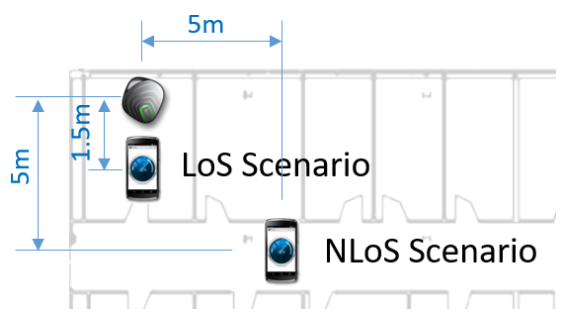

Fig. 6. Overview of the experimentation setup.

The magnitude of the Channel Impulse Responses (CIRs), i.e. $|\mathbf{H}(t, \tau)|$, is shown for both locations in Fig. 7. Each measurement (i.e. each row of the plot) contains 64 (complex valued) samples, hence we get $4800 \times 64=307200$ samples of the CIR. It is apparent not only that the delays are shorter in the LoS scenario (a) than in the NLoS scenario (b), but also that the total received energy is larger in the former case.

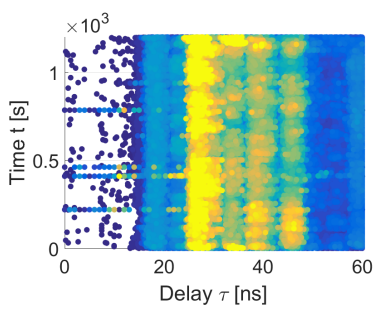

(a) $\operatorname{LoS}$

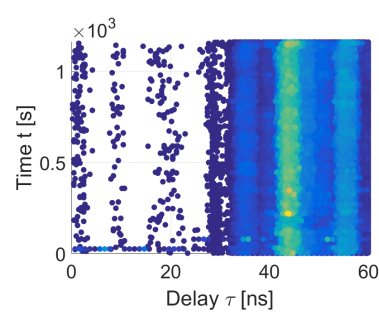

(b) $\mathrm{NLOS}$
Fig. 7. The magnitude of the channel impulse response $|\mathbf{H}(t, \tau)|$.

\section{A. TOA Measurements}

We start with the TOA measurement model (5) of Sec. II, where we assume the same characteristics for all BSs, and hence drop the corresponding subscript $i$. To estimate the parameters in (5), i.e. $\left[w_{r}, \mu_{r}, \sigma_{r}\right]$ for each MPC $r=1, \ldots, N_{r}$, we start by calculating the expected value of the energy with respect to the delay $\tau$, i.e. $E\{|\mathbf{H}(t, \tau)|, \tau\}$. In a next step, we normalize this to get the expected energy with respect to $\tau$

$$
p_{|\mathbf{H}|}(\tau)=\frac{E\{|\mathbf{H}(t, \tau)| \mid \tau\}}{\sum_{\tau} E\{|\mathbf{H}(t, \tau)| \mid \tau\}} .
$$

Thereafter, we fit one Gaussian distribution $\mathcal{N}\left(\mu_{r}, \sigma_{r}\right)$ with weight $w_{r}$ to each of the $N_{r}$ strongest MPCs of $p_{|\mathbf{H}|}(\tau)$, by means of Algorithm 1. 


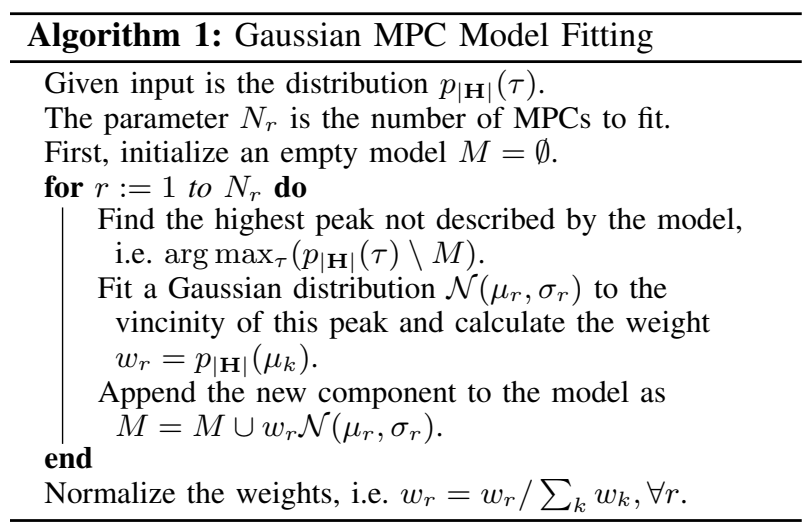

The results of this process is shown in Fig. 8, where both the original distribution (i.e. $\left.p_{|\mathbf{H}|}(\tau)\right)$ and the $N_{r}=$ 6 fitted Gaussian-modeled MPCs are shown for both scenarios. The numerical parameter values for (5) are given in Table I.

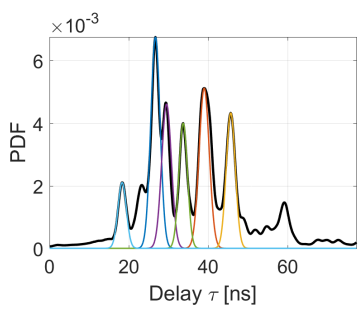

(a) $\operatorname{LoS}$

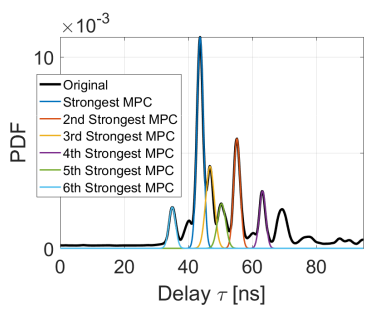

(b) NLoS
Fig. 8. The original distribution $p_{|\mathbf{H}|}(\tau)$, together with fitted weighted Gaussian distributions to the $N_{r}=6$ strongest MPCs.

TABLE I

Statistical Properties of the Fitted Distributions

\begin{tabular}{cc|cccccc} 
& & \multicolumn{7}{|c}{ MPC r } \\
& & 1 & 2 & 3 & 4 & 5 & 6 \\
\hline \multirow{3}{*}{ LoS } & Weight $w_{r}$ & 0.25 & 0.21 & 0.16 & 0.18 & 0.14 & 0.07 \\
& Mean $\mu_{r}$ & 26.7 & 39.0 & 45.7 & 29.5 & 33.7 & 18.3 \\
& Stdev $\sigma_{r}$ & 1.2 & 1.3 & 1.1 & 1.2 & 1.1 & 1.1 \\
\hline \multirow{4}{*}{ NLoS } & Weight $w_{r}$ & 0.36 & 0.19 & 0.19 & 0.10 & 0.10 & 0.07 \\
& Mean $\mu_{r}$ & 43.7 & 55.2 & 46.8 & 63.2 & 50.4 & 35.0 \\
& Stdev $\sigma_{r}$ & 1.0 & 1.0 & 1.3 & 1.0 & 1.3 & 1.0 \\
\hline
\end{tabular}

\section{B. Error Model for Simulation}

We now turn to Sec. IV, and the derivation of the distributions $p_{\tilde{E}}^{i}$ in (12) and $p_{\tilde{E}_{d+u}}^{i}$ in (14). We assume the error probabilities $P_{F A}=0.10$ and $P_{L D}=0.20$ respectively. The distributions are calculated numerically, and shown in Fig. 9.

These distributions will be used to generate different realizations of the measurement errors in (11) - (14). For example, in the LoS scenario using the RTT measurement procedure, the resulting channel measurements will be as per Fig. 10 .

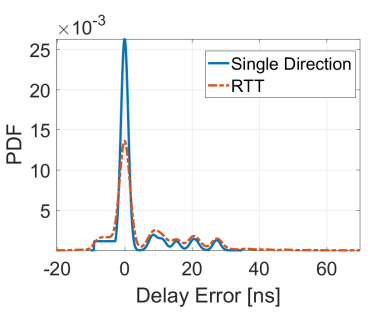

(a) $\operatorname{LoS}$

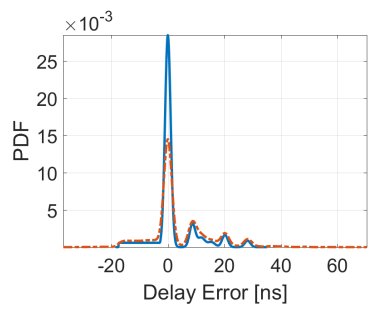

(c) NLoS

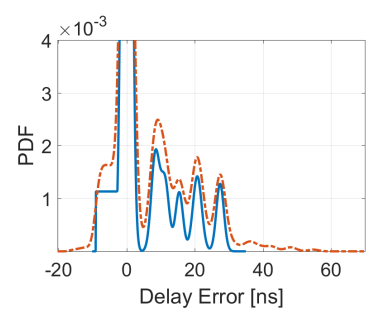

(b) LoS - Zoomed In

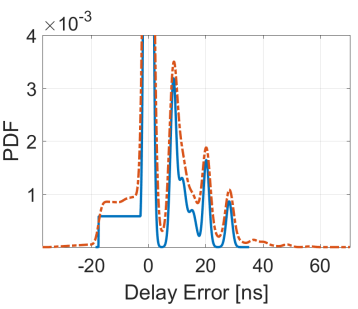

(d) NLoS - Zoomed In
Fig. 9. The TOA measurement error models of (12) and (14).

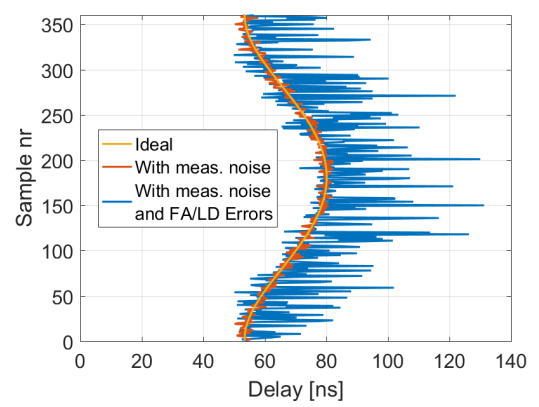

Fig. 10. RTT measurements in the LoS scenario, where both ideal measurements as well as noisy and erroneous measurements are shown.

\section{Error Model for Estimation}

In Sec. V we presented different assumptions the receiver may have on the measurement error. This was captured in four Models A-D, as given by (18) (21). The distributions are here calculated numerically, whereas an example of the LoS scenario using the RTT procedure is shown in Fig. 11. Please note that this figure is scaled for better visualization of the PDF.

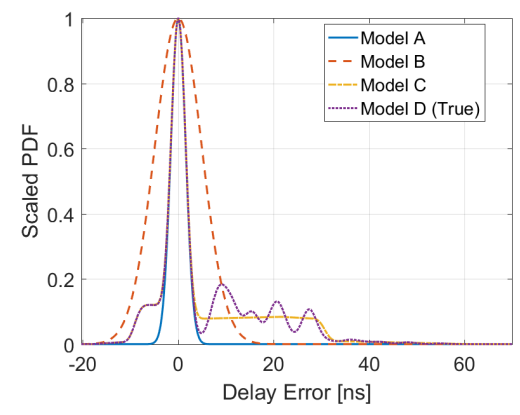

Fig. 11. The assumed error Models A-D given by (18)-(21).

As said earlier, these distributions will represent the expectation the receiver has on the measurement during the simulations in the following Sec. VII. 


\section{Simulation Results}

In the simulated scenario in Fig. 12, the UE moves at a constant angular velocity counter-clockwise in a circle of radius $2 \mathrm{~m}$ around the origin. The BS is at $[10,0]$.

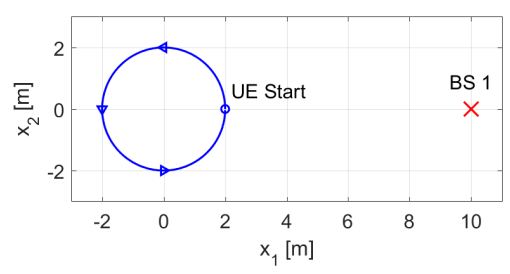

Fig. 12. Overview of the simulated scenario.

Each scenario (i.e. LoS/NLoS) is evaluated for each of the error Models A-D. Both an error free case $\left(P_{F A}=\right.$ $\left.P_{L D}=0\right)$ as well as a case with a rather large amount of FA and LD errors $\left(P_{F A}=0.1\right.$ and $\left.P_{L D}=0.2\right)$ are evaluated. The average results are derived from averaging over 40 Monte-Carlo (MC) simulations with different realizations in order to get good statistics.

\section{A. Results for Single-Direction Distance Estimation}

We start by estimating the delay for the singledirection measurements according to (16). An example of the measurements, the particles, the estimated distance as well as the true distance for one realization is shown in Fig. 13 for the error free case. The corresponding results, for the case with both FA and LD errors are shown in Fig. 14. We also plot a CDF of the Root Mean Square Error (RMSE) in Fig. 15 whereas the average RMSE is presented in Table II.

TABLE II

Average RMSE for Single-Direction Distance Estimation

\begin{tabular}{ll|cccc} 
& & \multicolumn{4}{|c}{ Average RMSE [m] } \\
& & Model A & Model B & Model C & Model D \\
\hline \multirow{2}{*}{ LoS } & Error Free & 0.18 & 0.39 & 0.19 & 0.19 \\
& FA and LD Errors & 0.45 & 1.63 & 0.21 & 0.20 \\
\hline \multirow{2}{*}{ NLoS } & Error Free & 0.17 & 0.36 & 0.17 & 0.17 \\
& FA and LD Errors & 0.50 & 1.27 & 0.19 & 0.18 \\
\hline
\end{tabular}

Based on these results, we see that that in the errorfree case, the Models A,C,D performs equally well and almost identically, whereas Model B is clearly much worse. This is due the fact that the (too) wide, underlying distribution of Model B captures too much of the noise in the measurements, i.e. gives a high probability also for the noisy measurements. This effect is further amplified in the case with FA and LD errors, where we in Fig. 14b see that the particles, and thus the estimate drifts off. We further see that the Models $\mathrm{C}$ and $\mathrm{D}$, performs very well in this situation. This since their underlying distributions well capture the actual measurement errors. It appears as

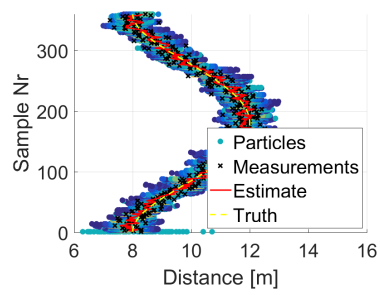

(a) Model A

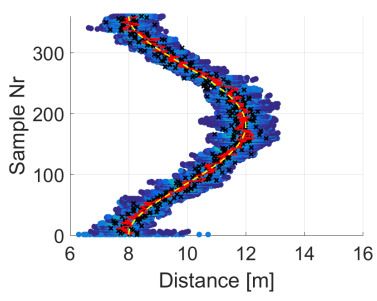

(c) Model C

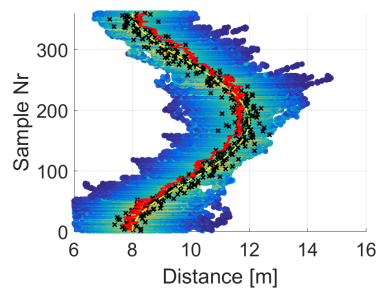

(b) Model B

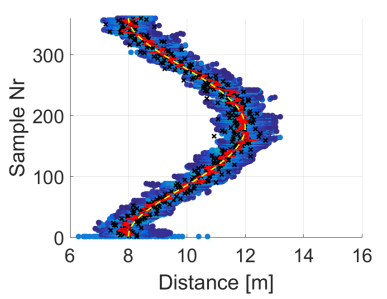

(d) Model D
Fig. 13. The particles, measurements and estimated as well as true distance for the LoS scenario using the single-direction estimation (16) for the Models A-D. Without FA and LD errors $\left(P_{F A}=P_{L D}=0\right)$.

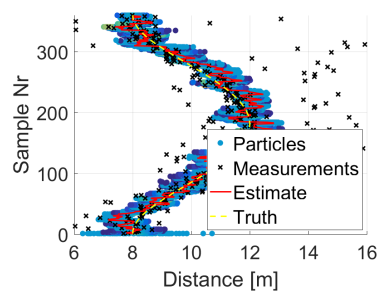

(a) Model A

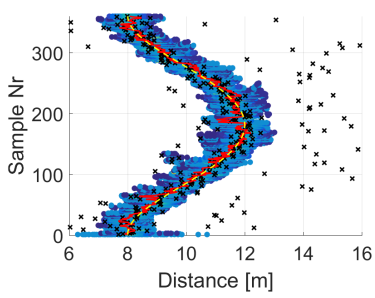

(c) Model C

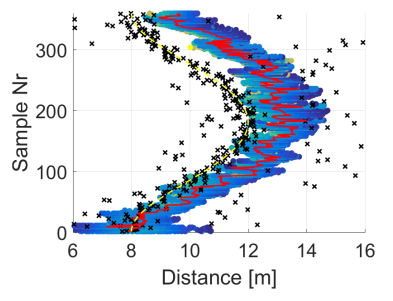

(b) Model B

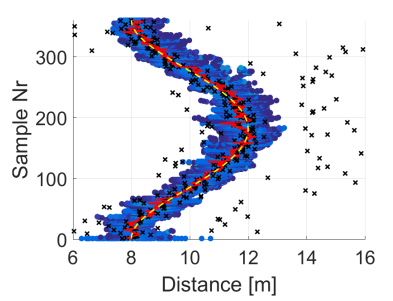

(d) Model D
Fig. 14. The particles, measurements and estimated as well as true distance for the LoS scenario using single-direction estimation (16) for the Models A-D. With FA and LD errors $\left(P_{F A}=0.1, P_{L D}=0.2\right)$.

if the simplified Model $\mathrm{C}$ is good enough. The interesting conclusion is that it seems not to be relevant to detect and manage other paths explicitly in the studied scenarios.

\section{B. Results for RTT-Based Distance Estimation}

Now, when RTT measurements according to (17) are used for estimation, we do not present particle plots as in Fig. 13 and 14. Instead we directly give the CDF of the RMSE in Fig. 16 and the average RMSE in Table III. The same conclusions as before still holds, i.e. Model B performs poorly, whereas the more informed models $\mathrm{C}$ and $\mathrm{D}$, provides significant gains in the FA and LD error case, but no drawback otherwise. Again, one conclusion 


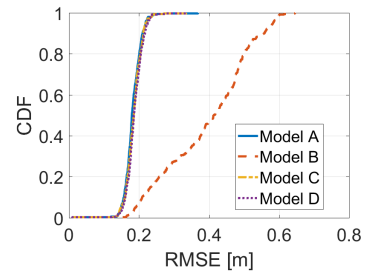

(a) LoS - No Error

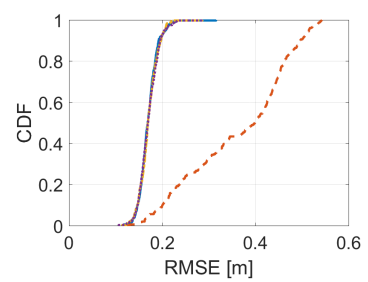

(c) NLoS - No Error

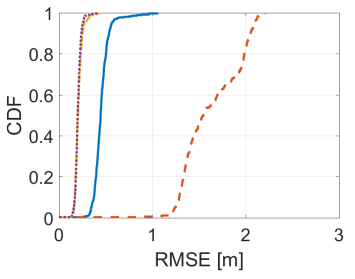

(b) LoS - with FA/LD Errors

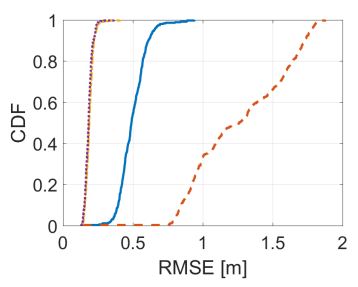

(d) NLoS - with FA/LD Errors
Fig. 15. CDF of the estimation error for the single-direction estimation.

is that it seems not to be relevant to detect and manage other paths explicitly in the studied scenarios. In the RTT case, that would involve including information about the additional paths in the exchange of information between the nodes. Given these results, information about the statistical properties is enough in these scenarios.

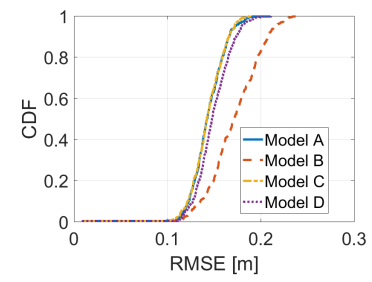

(a) LoS - No Error

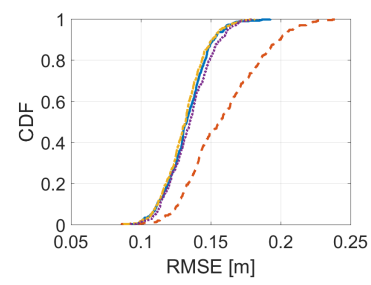

(c) NLoS - No Error

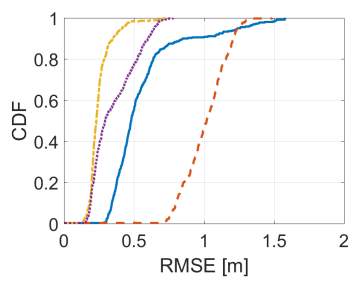

(b) LoS - with FA/LD Errors

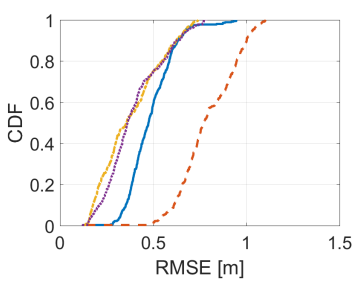

(d) NLoS - with FA/LD Errors
Fig. 16. CDF of the estimation error for the RTT-based estimation.

TABLE III

AvERAGE RMSE FOR RTT-BASED DistANCE Estimation

\begin{tabular}{ll|cccc} 
& & \multicolumn{4}{|c}{ Average RMSE [m] } \\
& & Model A & Model B & Model C & Model D \\
\hline \multirow{2}{*}{ LoS } & Error Free & 0.14 & 0.17 & 0.14 & 0.15 \\
& FA and LD Errors & 0.56 & 1.01 & 0.25 & 0.35 \\
\hline \multirow{2}{*}{ NLoS } & Error Free & 0.13 & 0.16 & 0.13 & 0.14 \\
& FA and LD Errors & 0.49 & 0.79 & 0.38 & 0.40 \\
\hline
\end{tabular}

\section{CONCLUSIONS}

Several positioning methods are based on TOA estimation, which can be subject to bias due to noise and non-line of sight propagation. In this paper we have investigated different TOA estimation error models for this, compared to genie knowledge of the TOA error distribution. The different models have been analyzed in the context of ranging, and the simulation models are based on data from a UWB measurement campaign. We conclude that more detailed information about the TOA error distribution implies significant ranging estimation improvement. However, very detailed information about individual paths does not improve the results compared to a simpler model of the error distribution in the studied scenarios.

\section{ACKNOWLEDGMENT}

This work was partially supported by the Wallenberg Autonomous Systems and Software Program (WASP).

\section{REFERENCES}

[1] K. Pahlavan, X. Li, and J. P. Makela, "Indoor geolocation science and technology," IEEE Communications Magazine, vol. 40, no. 2, pp. 112-118, Feb 2002.

[2] N. Patwari, J. N. Ash, S. Kyperountas, A. O. Hero, R. L. Moses, and N. S. Correal, "Locating the nodes: cooperative localization in wireless sensor networks," IEEE Signal Processing Magazine, vol. 22, no. 4, pp. 54-69, Jul. 2005.

[3] F. Gustafsson and F. Gunnarsson, "Mobile positioning using wireless networks: possibilities and fundamental limitations based on available wireless network measurements," IEEE Signal Processing Magazine, vol. 22, no. 4, pp. 41-53, Jul. 2005.

[4] S. Gezici, Z. Tian, G. B. Giannakis, H. Kobayashi, A. F. Molisch, H. V. Poor, and Z. Sahinoglu, "Localization via ultra-wideband radios: a look at positioning aspects for future sensor networks," IEEE Signal Processing Magazine, vol. 22, no. 4, pp. 70-84, Jul. 2005.

[5] J.-Y. Lee and R. A. Scholtz, "Ranging in a dense multipath environment using an UWB radio link," IEEE J. Sel. Areas Commun., vol. 20, no. 9, pp. 1677-1683, Dec. 2002.

[6] C. Falsi, D. Dardari, L. Mucchi, and M. Z. Win, "Time of arrival estimation for UWB localizers in realistic environments," EURASIP Journal on Advances in Signal Processing, vol. 2006, no. 1, 2006.

[7] D. Dardari, C. C. Chong, and M. Win, "Threshold-based timeof-arrival estimators in UWB dense multipath channels," IEEE Transactions on Communications, vol. 56, no. 8, pp. 1366-1378, August 2008.

[8] H. Ryden, A. A. Zaidi, S. M. Razavi, F. Gunnarsson, and I. Siomina, "Enhanced time of arrival estimation and quantization for positioning in LTE networks," in Proc. and Mobile Radio Communications (PIMRC) 2016 IEEE 27th Annual Int. Symp. Personal, Indoor, Sep. 2016.

[9] A. Mannesson, Joint Positioning and Multipath Radio Channel Estimation and Prediction. Department of Automatic Control, Lund Institute of Technology, Lund University, 2106.

[10] F. Gustafsson, Statistical sensor fusion. Studentlitteratur, 2010.

[11] I. Olofsson, "Enhancements in LTE OTDOA positioning for multipath environments," Master's thesis, Linköping University, Communication Systems, 2016.

[12] H. Nurminen, T. Ardeshiri, R. Piché, and F. Gustafsson, "Robust inference for state-space models with skewed measurement noise," IEEE Signal Processing Letters, vol. 22, no. 11, pp. 18981902, Nov. 2015.

[13] N. A. Alsindi, B. Alavi, and K. Pahlavan, "Measurement and modeling of ultrawideband TOA-based ranging in indoor multipath environments," IEEE Transactions on Vehicular Technology, vol. 58, no. 3, pp. 1046-1058, Mar. 2009.

[14] J. Khodjaev, Y. Park, and A. S. Malik, "Survey of NLOS identification and error mitigation problems in UWB-based positioning 
algorithms for dense environments," Annals of telecommunications - Annales des télécommunications, vol. 65, no. 5-6, pp. 301-311, aug 2009.

[15] S. Särkkä, Bayesian Filtering and Smoothing. Cambride University Press, 2013.

[16] BeSpoon, "Bespoon we position," April 2017. [Online]. Available: http://spoonphone.com/en/ 